\title{
PURE-INJECTIVES RELATIVE TO A COTORSION PAIR: APPLICATIONS
}

\author{
SERGIO ESTRADA \\ Departamento de Matemática Aplicada, Universidad de Murcia, Campus del Espinardo, \\ Espinardo (Murcia) 30100, Spain \\ e-mail: sestrada@um.es \\ and PEDRO A. GUIL ASENSIO \\ Departamento de Matemáticas, Universidad de Murcia, Campus del Espinardo, \\ Espinardo (Murcia) 30100, Spain \\ e-mail:paguil@um.es
}

(Received 31 March 2011; revised 2 May 2012; accepted 9 May 2012; first published online 2 August 2012)

\begin{abstract}
Finitely accessible categories naturally arise in the context of the classical theory of purity. In this paper we generalise the notion of purity for a more general class and introduce techniques to study such classes in terms of indecomposable pure injectives related to a new notion of purity. We apply our results in the study of the class of flat quasi-coherent sheaves on an arbitrary scheme.
\end{abstract}

2010 Mathematics Subject Classification. 18C35, 18A35, 18F20, 03 C98.

1. Introduction. In [5] Crawley-Boevey showed that finitely accessible additive categories admit a nice theory of purity (although he used the terminology of locally finitely presented additive for such categories). If $\mathcal{A}$ is a finitely accessible additive category, it is not difficult to prove that $\mathcal{A}$ is equivalent to the full subcategory $\operatorname{Flat}(S)$ of Mod-S of unitary flat right $S$-modules (i.e. modules such that $M \cdot S=M$ ) over a ring with enough idempotents (see [6, Theorem 1.1]). The pure-injective objects of Flat $(S)$ are precisely the flat cotorsion modules (where a right $R$-module $C$ is flat cotorsion if it is flat and does not have non-trivial extensions by any flat module, that is $\operatorname{Ext}_{S}^{1}(F, C)=0$ for all flat right $R$-module $\left.F\right)$. Therefore, the study of pureinjective objects in finitely accessible additive categories is equivalent to the study of flat cotorsion modules over rings with enough idempotents. The homological study of these modules was first considered by Harrison in [17] for abelian groups, and later on by Enochs in [8] for commutative Noetherian rings.

On the other hand, the class of flat cotorsion modules naturally arises when studying the so-called kernel of flat cotorsion pair. Cotorsion pairs $(\mathcal{F}, \mathcal{C})$ were first introduced by Salce [19] in 1979 in the category of abelian groups, but its definition can be easily extended to a more general setup. Then if $\mathcal{F}$ is the class of flat right $S$-modules and $\mathcal{C}$ the class of cotorsion right $S$-modules, it is easy to prove (see for example [13, Lemma 7.1.4]) that the pair $(\mathcal{F}, \mathcal{C})$ is a cotorsion pair known as the flat cotorsion pair and its kernel (the modules in the class $\mathcal{F} \cap \mathcal{C}$ ) is the class of flat

Both authors are supported by the DGI and by the Fundación Séneca. First author is also supported by the Junta de Andalucia. Part of the sources of both institutions come from the FEDER funds of the European Union. 
cotorsion modules. So in the language of cotorsion pairs, the study of pure-injective objects in finitely accessible additive categories is equivalent to the study of the kernel of the flat cotorsion pair over rings with enough idempotents.

This is a starting point of our paper. Our goal is to study 'pure-injectives' relative to the kernel of an arbitrary cotorsion pair in the Grothendieck category (so not just the flat cotorsion pair in Mod-S). Our approach has an advantage that can be applied to more general classes $\mathcal{F}$ of the Grothendieck category $\mathcal{A}$ (these need not be finitely accessible, or even $\lambda$-accessible, for $\lambda$ a regular cardinal). We will require $\mathcal{F}$ to be a Kaplansky class. This notion was introduced by Enochs and López Ramos in [14] and has been shown to be very fruitful in proving the existence of covers (and envelopes) with respect to class $\mathcal{F}$ in several algebraic categories, essentially by showing that $\mathcal{F}$ is a Kaplansky class (cf. $[3,4,9,10,15])$. Our main concern is to apply our results in categories of sheaves. Namely, apart from very particular schemes $X$ like non-singular irreducible curves over a field $k$, it is not known whether the class $\mathcal{F}$ of all flat quasicoherent sheaves on a general scheme $X$ is a finitely accessible additive category or not, so the general methods of purity theory cannot be applied to this setting. However, the class $\mathcal{F}$ of all flat quasi-coherent sheaves on an arbitrary scheme is always a Kaplansky class ([9, Theorem 4.1]), so the methods of this paper can be applied.

On the other hand, when $\mathcal{F}$ is a Kaplansky class, there is a canonical cotorsion pair $(\mathcal{F}, \mathcal{C})$, so one can also define 'pure-injectives' in this setup, and then use them to study structure of the class $\mathcal{F}$, just like pure-injective $R$-modules are used to study properties of Mod- $R$. For instance, Ziegler [22, Corollary 6.9] showed that there exist enough pure-injective indecomposable objects in the category Mod- $R$ in the sense that every module is elementarily equivalent to a direct product of pure-injective indecomposable modules. We will show in Theorem 4.1 that every object in the $\mathcal{F}$ can be purely embedded (in a sense that we will specify) into a direct pseudo-product of indecomposable objects in $\mathcal{F} \cap \mathcal{C}$ (the pure-injective objects of $\mathcal{F}$ ).

Again, our main motivation for developing a general theory of pure-injective objects relative to a Kaplansky class comes from the sheaf theory. In a recent paper [18, Section 3], Murfet and Salarian have stirred up interest of flat cotorsion quasicoherent sheaves in their study of a generalised homotopy category of totally acyclic complexes (over a semi-separated noetherian scheme). We hope that our results on indecomposable flat cotorsion quasi-coherent sheaves in Section 4 can help in understanding the structure of such complexes.

2. Background and notation. Throughout the paper $\mathcal{A}$ will be the Grothendieck category endowed with a faithful functor $U: \mathcal{A} \rightarrow$ Set, where Set denotes the category of sets (following Section 5 in [1], $\mathcal{A}$ is called construct). For instance, if $\mathcal{A}=\mathfrak{Q} \mathfrak{c o}(X)$, the category of quasi-coherent sheaves on scheme $X, U(F)=\sqcup_{v \in \mathcal{V}} F(v)$, where $\mathcal{V}$ is a fixed open affine cover of $X$.

By abuse of notation we write $x \in A$ instead of $x \in U(A)$ for any object $A$ in $\mathcal{A}$. Analogously $|A|$ will denote the cardinality of $U(A)$.

Now we will recall some basic notions on (pre)covers and (pre)envelopes that will be needed in the sequel. If $\mathcal{F}$ is a class of objects of $\mathcal{A}$, then an $\mathcal{F}$-precover of an object $X$ of $\mathcal{A}$ is a morphism $\varphi: F \rightarrow X$ where $F \in \mathcal{F}$ and $\operatorname{Hom}(G, F) \rightarrow \operatorname{Hom}(G, X)$ is surjective for all $G \in \mathcal{F}$. If furthermore any $f: F \rightarrow F$ with $\varphi \circ f=\varphi$ is an automorphism of $F$, then $\varphi$ is said to be an $\mathcal{F}$-cover of $X$ (clearly an $\mathcal{F}$-cover of $X$ is unique up to isomorphism if it exists). The dual notions are that of an $\mathcal{F}$-pre-envelope $\beta: X \rightarrow F$ and of an $\mathcal{F}$-envelope. 
For a class $\mathcal{F}$ of objects of $\mathcal{A}, \mathcal{F}^{\perp}$ will denote the class of objects $C$ of $\mathcal{A}$ such that $\operatorname{Ext}^{1}(F, C)=0$ for all $F \in \mathcal{F}$. Similarly, ${ }^{\perp} \mathcal{F}$ will denote the class of objects $D$ of $\mathcal{A}$ such that $\operatorname{Ext}^{1}(D, F)=0$ for all $F \in \mathcal{F}$.

Definition 2.1. (see [19] or [13, Chapter 7]). A pair of classes $(\mathcal{F}, \mathcal{C})$ of objects of $\mathcal{A}$ is said to be a cotorsion pair on $\mathcal{A}$ if $\mathcal{F}^{\perp}=\mathcal{C}$ and ${ }^{\perp} \mathcal{C}=\mathcal{F}$. A pair $(\mathcal{F}, \mathcal{C})$ is cogenerated by $\mathcal{S} \subseteq \mathcal{F}$ whenever $\mathcal{S}^{\perp}=\mathcal{C}$.

We note that if $(\mathcal{F}, \mathcal{C})$ is a cotorsion pair on $\mathcal{A}$, then $\mathcal{F}$ contains all the projective objects of $\mathcal{A}$ (there can be no other than the zero object) and $\mathcal{F}$ is closed under extensions, arbitrary direct sums and retracts. There is a dual claim for $\mathcal{C}$.

We also note that given an exact sequence

$$
0 \rightarrow C \rightarrow F \rightarrow X \rightarrow 0
$$

with $C \in \mathcal{C}, F \in \mathcal{F}$ where $(\mathcal{F}, \mathcal{C})$ is a cotorsion pair, we have the exact sequence

$$
\operatorname{Hom}(G, F) \rightarrow \operatorname{Hom}(G, X) \rightarrow \operatorname{Ext}^{1}(G, C)=0,
$$

for any $G \in \mathcal{F}$. Hence $F \rightarrow X$ is an $\mathcal{F}$-precover.

Definition 2.2. (see [21]). An $\mathcal{F}$-precover $F \rightarrow X$ is said to be a special $\mathcal{F}$-precover if $F \rightarrow X$ is part of an exact sequence

$$
0 \rightarrow C \rightarrow F \rightarrow X \rightarrow 0
$$

with $C \in \mathcal{F}^{\perp}$. A special $\mathcal{C}$-pre-envelope (for a class $\mathcal{C}$ ) is defined by the existence of an exact sequence

$$
0 \rightarrow X \rightarrow C \rightarrow F \rightarrow 0
$$

with $F \in{ }^{\perp} \mathcal{C}$.

Definition 2.3. A cotorsion pair $(\mathcal{F}, \mathcal{C})$ on $\mathcal{A}$ is said to have enough projectives (resp. enough injectives) if every object has a special $\mathcal{F}$-precover (resp. a special $\mathcal{C}$ pre-envelope). A cotorsion pair having enough injectives and projectives is called a complete cotorsion pair.

We recall the following:

Proposition 2.1. (Wakamatsu's lemma). If a class of objects $\mathcal{F}$ in $\mathcal{A}$ is closed under extensions and if $\varphi: F \rightarrow X$ is an $\mathcal{F}$-cover, then $\operatorname{ker}(\varphi) \in \mathcal{F}^{\perp}$.

Proof. See ([21, Lemma 2.1.1]) for a proof in a category of modules. The proof is easily modified to work in the Grothendieck category.

There is a dual result.

Proposition 2.2. If $\mathcal{C}$ is a class of objects closed under extensions and if $\alpha: X \rightarrow C$ is a $\mathcal{C}$-envelope, then $\operatorname{coker}(\alpha) \in{ }^{\perp} \mathcal{C}$.

Proof. By duality.

Definition 2.4. A cotorsion $\operatorname{pair}(\mathcal{F}, \mathcal{C})$ on $\mathcal{A}$ is said to be perfect if every object $X$ of $\mathcal{A}$ has an $\mathcal{F}$-cover and a $\mathcal{C}$-envelope. 
We note that if the class $\mathcal{F}$ contains a generator of $\mathcal{A}$, any $\mathcal{F}$-precover $\varphi: F \rightarrow X$ is an epimorphism. Similarly, any $\mathcal{C}$-pre-envelope $\alpha: X \rightarrow C$ is a monomorphism (because $\mathcal{C}$ contains all the injective objects of $\mathcal{A}$ ). Therefore, by Proposition 2.1, any perfect cotorsion pair $(\mathcal{F}, \mathcal{C})$ which contains a generator is complete.

Now we will define the notion of a Kaplansky class. As we shall see in the next section, there is a quite strong relation between Kaplansky classes and the existence of $\mathcal{F}$-covers and $\mathcal{F}^{\perp}$-envelopes.

Definition 2.5. A class $\mathcal{F}$ of objects of $\mathcal{A}$ is said to be a Kaplansky class (see [14]) of $\mathcal{A}$ if for each cardinal $\kappa$ there is a cardinal $\lambda$ such that if $S \subset F$ for some $F \in \mathcal{F}$ where $|S| \leq \kappa$ then there is an $F^{\prime} \subset F$ with $S \subset F^{\prime}$ where $\left|F^{\prime}\right| \leq \lambda$ and where $F^{\prime}$ and $F / F^{\prime}$ are both in $\mathcal{F}$.

EXAMPLE 2.1.

(1) If $\mathcal{A}=$ Mod- $R$, the class of all projective $R$-modules is Kaplansky (by the Kaplansky Theorem). This is standard and a motivating example for the definition.

(2) If $\mathcal{A}=\operatorname{Mod}-R$, then $\mathcal{F}=$ the class of all flat right $R$-modules is Kaplansky (apply [4, Lemma 1] to $\mathcal{F}$ ). More generally, if $\mathcal{F}$ is closed under pure submodules and pure quotients, then $\mathcal{F}$ is a Kaplansky class (see, for instance, [21, Lemma 2.5.2]).

(3) If $\mathcal{A}=\mathcal{O}_{X}$-Mod the category of sheaves of $\mathcal{O}_{X}$-modules, the class $\mathcal{F}$ of flat $\mathcal{O}_{X}$-modules is a Kaplansky class (apply [15, Proposition 2.4] to $\mathcal{F}$ ).

(4) If $\mathcal{A}=\mathfrak{Q} \mathfrak{c o}(X)$ the category of quasi-coherent sheaves on an arbitrary scheme $X$, the class $\mathcal{F}$ of flat quasi-coherent sheaves is Kaplansky (apply [9, Proposition 3.3] to $\mathcal{F}$ ).

(5) If $\mathcal{F}$ is a deconstructible class (see [20] for definitions) in the Grothendieck category $\mathcal{A}$, then $\mathcal{F}$ is a Kaplansky class (see [20, Corollary 2.7]).

(6) If $\mathcal{A}=\mathfrak{Q} \mathfrak{c o}(X)$ the category of quasi-coherent sheaves on an arbitrary scheme $X$, the class of (infinite-dimensional) vector bundles and the so-called (restricted) Drinfeld vector bundles are examples of Kaplansky classes (see [16, Corollaries 3.12 and 3.13]).

(7) If $\mathcal{A}$ is the Gorenstein category (see [10] for definitions) and $\mathcal{W}=$ the class of objects $W$ of $\mathcal{A}$ such that injdim $W \leq n$, then $\mathcal{W}$ is a Kaplansky class $([\mathbf{1 0}$, Corollary 2.13]).

(8) Let $\mathcal{A}$ be the Grothendieck category and $\mathbf{C}(\mathcal{A})$ the category of unbounded chain complexes in $\mathcal{A}$. Then, if $\mathcal{F}$ is a Kaplansky class closed under taking direct limits, the following classes in $\mathbf{C}(\mathcal{A})$ are also Kaplansky classes (see [20, Theorem 4.2, Corollary 2.7]):

(a) $\mathbf{C}(\mathcal{F})=\left\{\left(Y_{n}\right) \in \mathbf{C}(\mathcal{A}): Y_{n} \in \mathcal{F}, \forall n \in \mathbb{Z}\right\}$.

(b) $\widetilde{\mathcal{F}}=\left\{Y \in \mathbf{C}(\mathcal{A}): Y\right.$ is exact and $\left.\operatorname{ker}\left(Y_{n} \rightarrow Y_{n-1}\right) \in \mathcal{F}, \forall n \in \mathbb{Z}\right\}$.

(c) If $\mathcal{F}$ contains a generator of $\mathcal{A}$, the class dg- $\mathcal{F}$ of all chain complexes $Y \in \mathbf{C}(\mathcal{F})$ such that every chain complex morphism $Y \rightarrow C$ with $C \in \widetilde{\mathcal{F}^{\perp}}$ is nullhomotopic (where $\widetilde{\mathcal{F}}{ }^{\perp}$ is as in the previous item but with the class $\mathcal{F}^{\perp}$ instead of $\mathcal{F}$ ).

3. Kaplansky classes and perfect cotorsion pairs. In the next theorem we will prove that, under certain conditions, Kaplansky classes provide us examples of covers and envelopes with respect to them. 
THEOREM 3.1. Let $\mathcal{F}$ be a Kaplansky class of objects of $\mathcal{A}$, which is closed under direct sums, extensions and well ordered direct limits. Then the pair $\left(\mathcal{F}, \mathcal{F}^{\perp}\right)$ has enough injectives. If furthermore the generator of $\mathcal{A}$ is in $\mathcal{F}$, the pair $\left(\mathcal{F}, \mathcal{F}^{\perp}\right)$ is a perfect cotorsion pair.

Proof. Let $F \in \mathcal{F}$ and $x \in F$. Since $\mathcal{F}$ is Kaplansky, there is a cardinal $\lambda$ and $F_{0} \subseteq F$ with $x \in F_{0}$, where $\left|F_{0}\right| \leq \lambda$ and where $F_{0}, F / F_{0} \in \mathcal{F}$. Now, let us consider $x \in F \backslash F_{0}$. Then, as before, there exists $F_{1} / F_{0} \subseteq F / F_{0}$ with $x \in F_{1} \backslash F_{0}$, such that $F_{1} / F_{0}, F / F_{0} \in \mathcal{F}$ and $\left|F_{1} / F_{0}\right| \leq \lambda$. For the first limit ordinal $\omega$, we set $F_{\omega}=\cup_{\alpha<\omega} F_{\alpha} \in \mathcal{F}$, then, since $\mathcal{F}$ is closed under well-ordered direct limits, we infer that $F / F_{\omega} \in \mathcal{F}$, so we may continue the induction. Then, since any Grothendieck category is locally small, the process will eventually stop for some ordinal $\mu$. Therefore, we have expressed $F=\cup_{\alpha \leq \mu} F_{\alpha}$ such that $F_{0}, F_{\alpha+1} / F_{\alpha} \in \mathcal{F}$ and $\left|F_{0}\right|,\left|F_{\alpha+1} / F_{\alpha}\right| \leq \lambda$ for any successor ordinal $\alpha<\mu$. Now if we let $\mathcal{S} \subseteq \mathcal{F}$ be a set of representatives of $G \in \mathcal{F}$ with $|G| \leq \lambda$, we follow from Eklof's lemma (see [7, Theorem 1.2] $]^{1}$ ) that $\mathcal{S}^{\perp}=\mathcal{F}^{\perp}$, hence the pair $\left(\mathcal{F}, \mathcal{F}^{\perp}\right)$ is cogenerated by the set $\mathcal{S}$. Then Theorem 2.5 in [11] tells us that the pair $\left(\mathcal{F}, \mathcal{F}^{\perp}\right)$ has enough injectives.

Finally, let us see the last part of the statement. By Theorem 2.6 in [11] every object $M$ in $\mathcal{A}$ has an $\mathcal{F}$-cover and an $\mathcal{F}^{\perp}$-envelope. Now let $Y \in^{\perp} \mathcal{F}^{\perp}$, and $F \rightarrow Y \rightarrow 0$ be an $\mathcal{F}$-cover of $Y$. By Proposition 2.1, the sequence $0 \rightarrow \operatorname{ker}(F \rightarrow Y) \rightarrow F \rightarrow Y \rightarrow 0$ splits, so $Y$ is a retraction of $F$ and therefore $Y \in \mathcal{F}$. Hence, the pair $\left(\mathcal{F}, \mathcal{F}^{\perp}\right)$ is a perfect cotorsion pair.

4. Purity relative to a cotorsion pair. Let $\mathcal{A}=\operatorname{Mod}-R$. Then it is known that the right $R$-module $F$ is flat if and only if each short exact sequence $0 \rightarrow M \rightarrow N \rightarrow F \rightarrow 0$ in Mod- $R$ is pure. Flat modules are known to be the left part of the so-called flat cotorsion pair $(\mathcal{F}, \mathcal{C})$ (see [13, Lemma 7.1.4]). This justifies the following definition.

Definition 4.1. A short exact sequence $0 \rightarrow X \rightarrow Y \rightarrow Z \rightarrow 0$ in $\mathcal{A}$ is called pure relative to the cotorsion pair $(\mathcal{F}, \mathcal{C})$ (or $\mathcal{F}$-pure, for short) if $Z \in \mathcal{F}$. In particular, $X \subseteq Y$ is an $\mathcal{F}$-pure subobject whenever $0 \rightarrow X \hookrightarrow Y \rightarrow X / Y \rightarrow 0$ is $\mathcal{F}$-pure.

So we get the notion of a pure-injective object $M \in \mathcal{F}$ relative to the cotorsion pair $(\mathcal{F}, \mathcal{C})$ whenever each exact sequence $0 \rightarrow M \rightarrow Y \rightarrow Z \rightarrow 0$, which is pure relative to $(\mathcal{F}, \mathcal{C})$, splits. Then it is immediate to observe that pure-injectives in $\mathcal{F}$ relative to the cotorsion pair do coincide with objects in the class $\mathcal{F} \cap \mathcal{C}$. This class is often known as the kernel of the cotorsion pair. Of course, when $\mathcal{F}$ is the class of flat right $R$-modules, then the pure-injectives in $\mathcal{F}$ relative to the flat cotorsion pair are the usual pure-injectives in Flat $(R)$, that is the class of flat cotorsion right $R$-modules.

\subsection{Pseudo-products on abelian categories.}

Definition 4.2. Let $\mathcal{A}$ be an abelian category and $\left\{A_{i}\right\}_{i \in I}$ be a family of objects of $\mathcal{A}$. A pseudo-product of the family $\left\{A_{i}: i \in I\right\}$ is an object $A \in \mathcal{A}$ and a family of morphisms $\left\{q_{i}: A \rightarrow A_{i}\right\}_{i \in I}$ that verifies the following: If $B$ is an object of $\mathcal{A}$ and $\left\{f_{i}: B \rightarrow A_{i}\right\}_{i \in I}$ is a family of morphisms, then there exists a morphism $\varphi: B \rightarrow A$ such that $q_{i} \circ \varphi=f_{i}, \forall i \in I$.

\footnotetext{
${ }^{1}$ The arguments in [7, Theorem 1.2] are for modules, but easily carried over for the Grothendieck category.
} 
4.2. Main theorem. For the next lemma we will assume that $\mathcal{F}$ is a Kaplansky class that satisfies all conditions of Theorem 3.1 (so the pair $\left(\mathcal{F}, \mathcal{F}^{\perp}\right.$ ) is a perfect cotorsion pair).

LEMMA 4.1. Given a cardinal $\kappa$ there is a cardinal $\gamma$ such that if $|M| \leq \kappa$ then $|C(M)| \leq \gamma\left(\right.$ where $C(M)$ is the $\mathcal{F}^{\perp}$-envelope of $\left.M\right)$.

Proof. Firstly we note that it suffices to prove that the statement is true for some $\mathcal{F}^{\perp}$-pre-envelope $M \rightarrow C$ of $M$ because $\mathcal{F}^{\perp}$-envelopes are retracts of $\mathcal{F}^{\perp}$-pre-envelopes (see [21, Proposition 1.2.1]). By the proof of Theorem 2.5 in [12], for any ordinal $\beta$ there exists a continuous chain $\left(M_{\alpha}\right)_{\alpha \leq \beta}$ of objects, where $M_{0}=M$, such that $M_{\alpha+1} / M_{\alpha} \in \mathcal{F}$ for every ordinal $\alpha+1<\beta$ and $M_{\gamma}=\cup_{\alpha<\gamma} M_{\alpha}$ whenever $\gamma$ is a limit ordinal. So we can find a family $\left(\kappa_{\alpha}\right)_{\alpha \leq \beta}$ of cardinal numbers such that $\kappa_{0}=\kappa$ and $|M|=\left|M_{0}\right| \leq \kappa$ and $\left|M_{\alpha}\right| \leq \kappa_{\alpha}$ for each $\alpha \leq \beta$. Now we choose the ordinal $\mu$ attained to $\kappa$, as in [12, Proposition 2.3]. Then again, by the argument given in the proof of Theorem 2.5 in [12], $C=\cup_{\alpha \leq \mu} M_{\alpha} \in \mathcal{F}^{\perp},|C| \leq \kappa_{\mu}$ and $0 \rightarrow M \rightarrow C$ is an $\mathcal{F}^{\perp}$-pre-envelope (in fact, a special $\mathcal{F}$-pre-envelope).

LEMMA 4.2. Let $\mathcal{F}$ be a Kaplansky class closed under direct sums, extensions and well ordered direct limits. There exists an infinite cardinal $\delta$ such that $|C| \leq \delta$, for all indecomposable $C \in \mathcal{F} \cap \mathcal{F}^{\perp}$.

Proof. We will show that there exists a cardinal $\lambda$ such that, up to isomorphism, every indecomposable $C \in \mathcal{F} \cap \mathcal{F}^{\perp}$ is the cotorsion envelope of an object $S \in \mathcal{F}$ with $|S| \leq \lambda$. Therefore, the result will follow in view of Lemma 4.1. Let $C \in \mathcal{F} \cap \mathcal{F}^{\perp}$ be indecomposable and $0 \neq x \in C$. Since $\mathcal{F}$ is a Kaplansky class, there is a cardinal $\lambda$ and there is $F \in \mathcal{F}$ such that $x \in F \subseteq C,|F| \leq \lambda$ and $C / F \in \mathcal{F}$. So $F \rightarrow C$ is an $\mathcal{F}^{\perp}$-preenvelope of $C$. Now let $C(F)$ be the $\mathcal{F}^{\perp}$-envelope of $F$. It follows that $C(F)$ is a retract of $C$, and since $C$ is indecomposable, $C=C(F)$.

THEOREM 4.1. Let $\mathcal{F}$ be a Kaplansky class in $\mathcal{A}$ which is closed under direct sums, extensions and well-ordered direct limits, and such that $\mathcal{F}$ contains a generator of $\mathcal{A}$. Then every $F \in \mathcal{F}$ is an $\mathcal{F}$-pure sub-object of a pseudo-product of a family of indecomposable objects in $\mathcal{F} \cap \mathcal{F}^{\perp}$. Such pseudo-product can be chosen to be a special $\mathcal{F}^{\perp}$-pre-envelope of $F$.

Proof. Let $F \in \mathcal{F}$ and let $0 \neq x \in F$. Let us consider

$$
\Sigma=\left\{F^{\prime} \subseteq F: F^{\prime}, F / F^{\prime} \in \mathcal{F} \text { and } x \notin F^{\prime}\right\}
$$

It is clear that $\Sigma \neq \emptyset$ (because the zero object is in $\mathcal{F}$ ). Now let $\left\{F_{\alpha}\right\}_{\alpha \in \Lambda}$ be a non-empty totally ordered subset of $\Sigma$. We have to show that $\left\{F_{\alpha}\right\}_{\alpha \in \Lambda}$ has an upper bound $F^{\prime \prime}$ in $\Sigma$. Let us define $F^{\prime \prime}=\cup_{\alpha \in \Lambda} F_{\alpha} \subseteq F$. Since $\mathcal{F}$ is closed under well-ordered direct limits, it follows that both $F^{\prime \prime}$ and $F / F^{\prime \prime}=\lim _{\alpha \in \Lambda} F / F_{\alpha}$ are in $\mathcal{F}$. Finally, since for each $\alpha \in \Lambda x \notin F_{\alpha}$, we infer that $F^{\prime \prime} \in \Sigma$. Therefore, by Zorn's lemma, there is a maximal element $F_{0} \in \mathcal{F}$ of $\Sigma$. Then $F / F_{0} \neq 0$ (because $x \notin F_{0}$ ) and it is indecomposable. For if $F / F_{0}=A \oplus B$ then w.l.o.g. we may assume that the composition map

$$
F \stackrel{\pi}{\rightarrow} A \oplus B \stackrel{\rho}{\rightarrow} A
$$


(where $\pi$ and $\rho$ are canonical epimorphisms) is such that $\operatorname{ker}(\rho \circ \pi) \supseteq F_{0}, A=$ $F / \operatorname{ker}(\rho \circ \pi) \in \mathcal{F}, \operatorname{ker}(\rho \circ \pi) \in \mathcal{F}$ (because $\mathcal{F}$ is closed under extensions) and $x \notin$ $\operatorname{ker}(\rho \circ \pi)$, which is a contradiction with the maximality of $F_{0}$.

Now let us consider the canonical projection $\pi_{0}: F \rightarrow F / F_{0}$, so $\pi_{0}(x) \neq 0$ and let $u_{0}: F / F_{0} \rightarrow C\left(F / F_{0}\right)$ be the $\mathcal{F}^{\perp}$-envelope of $F / F_{0}$. Then $C\left(F / F_{0}\right) \in \mathcal{F} \cap \mathcal{F}^{\perp}$ (but it does not have to be indecomposable in general). Let us repeat the procedure with the element $u_{0} \pi_{0}(x)$ and with $F_{1}=C\left(F / F_{0}\right)$, so we get $F_{1}^{\prime} \subseteq F_{1}$ such that $F_{1} / F_{1}^{\prime}$ is indecomposable and take $F_{2}=C\left(F_{1} / F_{1}^{\prime}\right)$ and follow with this procedure. But by Lemma 4.1 this process must stop, so for each $0 \neq x \in F$ there will exist an indecomposable $U_{x} \in \mathcal{F} \cap \mathcal{F}^{\perp}$ and a map $\pi_{x}: F \rightarrow U_{x}$ such that $\pi_{x}(x) \neq 0$. Now let us consider the induced monomorphism $\pi=\left(\pi_{x}\right)_{x \in F}: F \rightarrow \prod_{x \in F} U_{x}$ that maps $y \mapsto\left(\pi_{x}(y)\right)_{x \in F}$. Let us take a short exact sequence

$$
0 \rightarrow F \stackrel{\pi}{\longrightarrow} \prod_{x \in F} U_{x} \rightarrow \operatorname{coker}(\pi) \rightarrow 0
$$

and consider the following commutative diagram

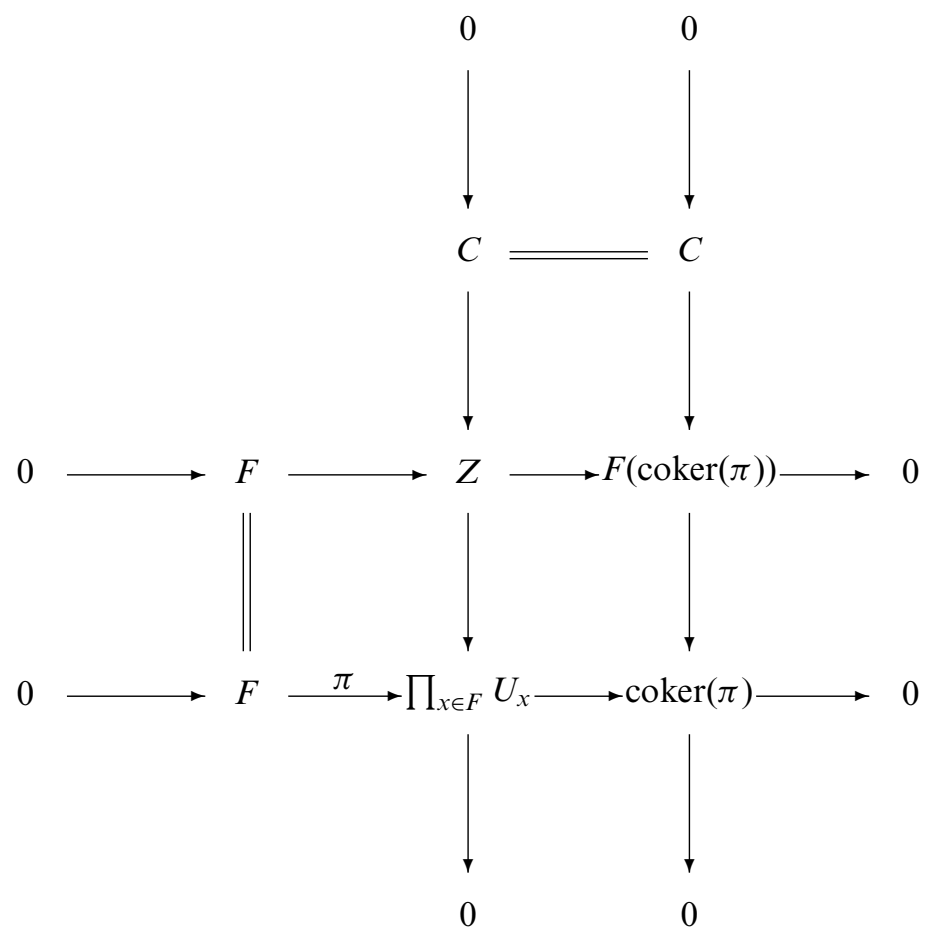

where $F(\operatorname{coker}(\pi))$ is the $\mathcal{F}$-cover of $\operatorname{coker}(\pi)$. Then $Z \in \mathcal{F}$ and $Z \rightarrow \prod_{x \in F} U_{x} \rightarrow 0$ is an $\mathcal{F}$-precover (since $C \in \mathcal{F}^{\perp}$ ), which is the desired pseudo-product of the family $\left\{U_{x}: x \in F\right\}$.

Definition 4.3. Let $\left\{U_{i}: i \in I\right\}$ be a family of indecomposable objects in $\mathcal{F} \cap \mathcal{F}^{\perp}$. We shall denote by $\prod_{i \in I}^{q} U_{i}$ a pseudo-product of the family $\left\{U_{i}: i \in I\right\}$. 
COROLlaRY 4.1. Let $\mathcal{F}$ be a Kaplansky class closed under direct sums, extensions and well-ordered direct limits. Let us consider the class

$$
\Omega=\left\{\text { indecomposable objects in } \mathcal{F} \cap \mathcal{F}^{\perp}\right\}
$$

Then

(1) $\Omega$ is a non-empty set.

(2) Given $F \in \mathcal{F}$, there exists a family of indecomposables $\left\{U_{i}: i \in I\right\} \subseteq \Omega$ such that $F$ is $\mathcal{F}$-pure in $\prod_{i \in I}^{q} U_{i}$.

5. Applications in categories of sheaves. In the present section we focus on the category $\mathfrak{Q} \mathfrak{c o}(X)$ of quasi-coherent sheaves on scheme $X$. We will see that in this case we can get a refinement of Theorem 4.1. In order to show this, we need to recall some known facts concerning this category. An object $M \in \mathcal{A}(\mathcal{A}$ is any category) is called $\kappa$-presentable for some regular cardinal $\kappa$ if $\operatorname{Hom}_{\mathcal{A}}(M,-)$ preserves $\kappa$-directed colimits. Then $\mathcal{A}$ is said to be locally $\kappa$-presentable (cf. [2, Definition 1.17]) provided that it is co-complete, and has a set of $\kappa$-presentable objects such that every object is a $\kappa$-directed colimit of them. The category $\mathcal{A}$ is locally presentable if it is locally $\kappa$-presentable for some regular cardinal $\kappa$.

THEOREM 5.1. For any scheme $X, \mathfrak{Q c o}(X)$ is locally presentable.

Proof. See Corollary 3.5 in [9].

A quasi-coherent sheaf $F$ is flat if $F \otimes_{\mathcal{R}}-$ is exact, where $\mathcal{R}$ denotes the structure sheaf of $X$. If $X=\operatorname{Spec}(A)$ for some commutative $\operatorname{ring} A$, then a short exact sequence

$$
0 \rightarrow \widetilde{M} \rightarrow \widetilde{N} \rightarrow \widetilde{L} \rightarrow 0
$$

in $\mathfrak{Q} \mathfrak{c o}(X)$ is pure whenever the corresponding short exact sequence of $A$-modules, $0 \rightarrow M \rightarrow N \rightarrow L \rightarrow 0$ is pure. Then a short exact sequence $0 \rightarrow T \rightarrow D \rightarrow P \rightarrow$ 0 in $\mathfrak{Q} \mathfrak{c o}(X)$ is pure if the corresponding short exact sequence $\left.\left.0 \rightarrow T\right|_{U} \rightarrow D\right|_{U} \rightarrow$ $\left.P\right|_{U} \rightarrow 0$ is pure in $\mathfrak{Q} \mathfrak{c o}(U)$ for each affine inclusion $U \hookrightarrow X$. Then $T \subseteq D$ is a pure quasi-coherent subsheaf whenever $0 \rightarrow T \rightarrow D \rightarrow D / T \rightarrow 0$ is pure in $\mathfrak{Q c o}(X)$. Let us consider the class $\mathcal{F}$ of all flat quasi-coherent sheaves on $\mathfrak{Q} \mathfrak{c o}(X)$. By Example 2.1(4) we know that it is a Kaplansky class. We point that in this case $\mathcal{F}^{\perp}$ is known as a class of cotorsion quasi-coherent sheaves.

THEOREM 5.2. Let $X$ be any scheme, then each quasi-coherent sheaf in $\mathfrak{Q} \mathfrak{c o}(X)$ has a flat cover and a cotorsion envelope.

Proof. See Corollary 4.2 in [9].

COROLlary 5.1. Each flat $F \in \mathfrak{Q} \mathfrak{c o}(X)$ is a pure quasi-coherent sub-sheaf of a flat cover of a product of indecomposable flat cotorsion quasi-coherent sheaves (so in particular $F$ is a pure quasi-coherent sub-sheaf of a pseudo-product of sheaves in $\mathcal{F}$ ).

Proof. Let $\mathcal{S}=\left\{S_{i}: i \in I\right\}$ be a set of $\lambda$-presentable generators of $\mathfrak{Q} \mathfrak{c o}(X)$ (assuming that $\mathfrak{Q} \mathfrak{c o}(X)$ is locally $\lambda$-presentable, with $\lambda$ being an infinite regular cardinal). Let $F \in \mathcal{F}$ and fix $S_{i} \in \mathcal{S}$. Given $0 \neq a \in F \otimes_{\mathcal{R}} S_{i}$ by the same arguments of the proof of Theorem 4.1, there exists a flat cotorsion quasi-coherent sheaf $U_{a, S_{i}}$ and a map $\pi_{a, S_{i}}: F \otimes_{\mathcal{R}} S_{i} \rightarrow U_{a, S_{i}} \otimes_{\mathcal{R}} S_{i}$ such that $\pi_{a, S_{i}}(a) \neq 0$. Then following the same 
steps as that in the proof of Theorem 4.1, we get a monomorphism

$$
\pi: F \otimes_{\mathcal{R}} S_{i} \longrightarrow \prod_{\substack { a \in F \\
\begin{subarray}{c}{a \\
S_{i} \in \mathcal{S}{ a \in F \\
\begin{subarray} { c } { a \\
S _ { i } \in \mathcal { S } } }\end{subarray}} U_{a, S_{i}} \otimes_{\mathcal{R}} S_{i}
$$

Now let $\mathcal{R}$ be the structure sheaf of scheme $X$. Then $\mathcal{R}=\lim _{\alpha<\lambda} S_{\alpha}$. Now using the fact that $F \otimes_{\mathcal{R}} \mathcal{R} \cong F$, the tensor product commutes with direct limits and $\lim _{\rightarrow}$ is exact, we follow that the induced map

$$
\pi^{\prime}: F \longrightarrow \prod_{\substack{a \in F \otimes_{\mathcal{R}} S_{i} \\ S_{i} \in \mathcal{S}}} U_{a, S_{i}}
$$

is a monomorphism, which is pure.

Now take the commutative square

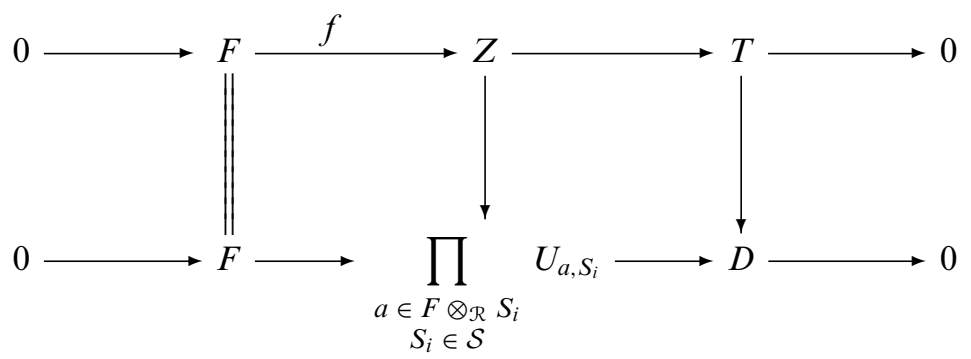

where $Z$ is a flat cover of $\prod U_{a, S_{i}}$ and $f: F \rightarrow Z$ is a morphism given by the definition of flat cover. Then, since $\pi^{\prime}$ is pure, it is immediate that $f$ is also pure.

REMARK 5.1.

(1) In Corollary 5.1 we do not require any condition on the scheme. Namely, we do not assume that $\mathcal{F}$ contains a generator of $\mathfrak{Q} \mathfrak{c o}(X)$.

(2) If $X$ is a non-singular irreducible curve or surface over a field, the class $\mathcal{F}$ is known to be a locally finitely accessible category. In this case the family of classical vector bundles completely determines the class $\mathcal{F}$, in the sense that every flat sheaf on $X$ is a directed colimit of these. For more general schemes it seems unlikely we can find such a family for $\mathcal{F}$, so Corollary 5.1 at least provides some light in understanding the structure of flat sheaves for the general case, in terms of indecomposable flat cotorsion sheaves. There is a full classification of flat cotorsion quasi-coherent sheaves in case $X=\mathbf{P}^{\mathbf{1}}(k)$ in [11].

\section{REFERENCES}

1. J. Adámek, H. Herrlich and G. E. Strecker, Abstract and concrete categories. The joy of cats, Repr. Theory Appl. Categ. 17 (2006), 1-507.

2. J. Adámek and J. Rosický, Locally presentable and accessible categories, vol. 189 (Cambridge University Press, Cambridge, UK, 1994).

3. S. T. Aldrich, E. Enochs, J. R. García-Rozas and L. Oyonarte, Covers and envelopes in Grothendieck categories. Flat covers of complexes with applications, J. Algebra 243 (2001), 615-630. 
4. L. Bican, R. El Bashir and E. Enochs, All modules have flat covers, Bull. Lond. Math. Soc. 33(4) (2001), 385-390.

5. W. W. Crawley-Boevey, Locally finitely presented additive categories, Comm. Algebra 22 (1994), 1641-1674.

6. N. V. Dung and J. L. García, Additive categories of locally finite representation type, J. Algebra 238 (2001), 200-238. $207-225$

7. P. C. Eklof, Homological algebra and set theory, Trans. Amer. Math. Soc. 227 (1977),

8. E. Enochs, Flat covers and flat cotorsion modules, Proc. Amer. Math. Soc. 97 (1984), $179-184$.

9. E. Enochs and S. Estrada, Relative homological algebra in the category of quasicoherent sheaves, Adv. Math. 194 (2005), 284-295.

10. E. Enochs, S. Estrada and J. R. García-Rozas, Gorenstein categories and Tate cohomology on projective schemes, Math. Nachr. 281 (2008), 525-540.

11. E. Enochs, S. Estrada, J. R. García Rozas and L. Oyonarte, Flat and cotorsion quasicoherent sheaves. Applications. Algebra Represent. Theory 7 (2004), 441-456.

12. E. Enochs, S. Estrada, J. R. García Rozas and L. Oyonarte, Flat covers in the category of quasi-coherent sheaves over the projective line, Comm. Algebra 32 (2004), 1497-1508.

13. E. Enochs and O. M. G. Jenda, Relative homological algebra, GEM 30 ( 2000) (W. de Gruyter, Berlin, Germany).

14. E. Enochs and J. A. López Ramos, Kaplansky classes, Rend. Sem. Mat. Univ. Padova 107 (2002), 67-79.

15. E. Enochs and L. Oyonarte, Flat covers and cotorsion envelopes of sheaves, Proc. Amer. Math. Soc. 130 (2001), 1285-1292.

16. S. Estrada, P. Guil Asensio, M. Prest and J. Trlifaj, Model category structures arising from Drinfeld vector bundles, arXiv: 0906.5213v1.

17. D. K. Harrison, Infinite abelian groups and homological methods, Ann. Math. 69(1959), 366-391.

18. D. Murfet and S. Salarian, Totally acyclic complexes over noetherian schemes, $A d v$. Math. 296 (2011), 1096-1133.

19. L. Salce, Cotorsion theories for abelian groups, Symp. Math. 23 (1979), 11-32.

20. J. Šłovíček, Deconstructibility and the Hill lemma in Grothendieck categories, Forum Math. (to appear).

21. J. Xu, Flat covers of modules (Lecture Notes in Mathematics, no. 1634 (Springer-Verlag, Berlin, Germany 1996).

22. M. Ziegler. Model theory of modules, Ann. Pure Appl. Log. 26(2) (1984), 149-213. 